\title{
ABELIAN TORSION GROUPS HAVING A MINIMAL SYSTEM OF GENERATORS $\left({ }^{1}\right)$
}

\author{
BY
}

\author{
SAMIR A. KHABBAZ
}

Notation AND TERMINology. Let $T$ denote an arbitrary Abelian torsion group. Let $G$ denote an arbitrary primary $p$-group to be considered fixed in each separate proposition. Let the symbol "iff" mean "if and only if," $<$ mean properly contained in, $\subset$ mean contained in, $N \backslash M$ mean the set of elements in $N$ and not in $M$, $\cong$ mean isomorphic to, $\left(N_{a}\right)_{a \in A}$ denote a family of sets, elements or groups-as the case may be $-N_{a}$ indexed by members of an index set $A$; and if for each $a \in A N_{a}$ is a group let $\oplus_{a \in A} N_{a}$ denote the direct sum of the $N_{a}$ 's $-\oplus$ denotes a direct sum. If $x \in G$ let $h(x)$ denote the ordinary height of $x$-see [2].

If $S$ is a subset or subgroup of $T$, let $|S|$ denote the power-cardinal number-of $S$. Let $(S)$ mean the same thing as $S$, and $\{S\}$ mean the subgroup generated by the elements of $S$. If $x_{1}, x_{2}, x_{3}, \cdots \in T$, let $\left(x_{1}, x_{2}, x_{3}, \cdots\right)$ denote the set whose only elements are $x_{1}, x_{2}, x_{3}, \cdots$, and let $\left\{x_{1}, x_{2}, x_{3}, \cdots\right\}$ $=\left\{\left(x_{1}, x_{2}, x_{3}, \cdots\right)\right\}$.

Definition. A subset $S$ of elements of $T$ is said to be a minimal system of generators of $T$-abbreviated by (m.s.g.)-iff $S$ generates $T$, and no proper subset of $S$ generates $T$.

Definition. A subset $S$ of elements of $T$ is said to be $a$ minimum system of generators of $T$-abbreviated by (M.s.g.) -iff $S$ generates $T$, and for every positive integer $n(1 \leqq n \leqq|S|)$ no $n$ elements of $S$ can be replaced by fewer than $n$ elements of $T$ in such a way that the resulting set still generates $T$.

\section{On primary groups.}

REMARK. Since most of the following propositions are trivially true for finite groups, we shall of ten assume while proving them that $G$ is infinite.

Lemma 1. Let $G$ have a (m.s.g.) $S$, and let $x \in S$. Then $h(x)=0$.

Proof. Assume for some $x \in S \exists y \in G$ such that $p y=x$. Then $\exists x_{1}, x_{2}, \cdots$, $x_{m} \in S$ and integers $n_{1}, \cdots, n_{m}$ such that $y=n_{1} x_{1}+\cdots+n_{m} x_{m}$. By the minimality of $S$ we may assume that $x_{1}=x$ and $n_{1} \neq 0$. Then $x=p y=n_{1} p x$ $+n_{2} p x_{2}+\cdots+n_{m} p x_{m}$. Thus $(S \backslash(x)) \cup(p x)$ is a (m.s.g.) of $G$. Continuing this with $p^{i+1} x$ in place of $p^{i} x$ we arrive finally at $(S \backslash(x)) \cup\left(p^{n} x\right)$ is a (m.s.g.) of $G$ where $p^{n} x=0$, which is impossible.

Presented to the Society, January 29, 1960 under the title Abelian groups with minimal systems of generators. Preliminary report; received by the editors March 8, 1960.

(1) This paper constitutes the second part of the author's doctoral dissertation submitted to the University of Kansas. 
Lemma 2. Let $G$ have a (m.s.g.) S. Suppose that $x=n_{1} x_{1}+\cdots+n_{m} x_{m}$, where $x_{1}, \cdots, x_{m} \in S$ and for some integer $i, 1 \leqq i \leqq m,\left(n_{i}, p\right)=1$. Then $\left(S \backslash\left(x_{i}\right)\right) \cup(x)$ is a (m.s.g.) of $G$.

Proof. We may take $i=1$. That $\left(S \backslash\left(x_{1}\right)\right) \cup(x)$ generates $G$ is trivial. To show it is minimal it suffices to show that no $x_{i}$ for $1<i \leqq m$ is a linear combination of elements of $\left(S \backslash\left(x_{1}, x_{i}\right)\right) \cup(x)$. So assume one such $x_{i}$, say $x_{m}$, is a linear combination of the elements of $\left(S \backslash\left(x_{1}, x_{m}\right)\right) \cup(x)$. Then $\exists$ elements $y_{m}, \cdots, y_{k}$ of $S$ different from the $x_{i}$ 's and integers $l_{1}, \cdots, l_{k}$, such that $x_{m}=l_{1} x+l_{2} x_{2}+\cdots+l_{m-1} x_{m-1}+l_{m} y_{m}+\cdots+l_{k} y_{k}$. Thus $x_{m}=l_{1} n_{1} x_{1}$ $+\left(l_{1} n_{2}+l_{2}\right) x_{2}+\left(l_{1} n_{3}+n_{3}\right) x_{3}+\cdots+\left(l_{1} n_{m-1}+l_{m-1}\right) x_{m-1}+l_{1} n_{m} x_{m}+l_{m} y_{m}+\cdots$ $+l_{k} y_{k}$. Since $S$ is minimal, the coefficients of all the $x$ 's and $y$ 's on the right hand side of the last equation which are not equal to $x_{m}$ must be multiples of $p$. In particular, $l_{1} n_{1}$ is a multiple of $p$. But $n_{1}$ was assumed to be relatively prime to $p$. Hence $l_{1}$ is a multiple of $p$. Hence also the coefficient $l_{1} n_{m}$ of $x_{m}$ is a multiple of $p$. Thus $h\left(x_{m}\right)>0$, contrary to Lemma 1 .

Lemma 3. Let $G$ have a (m.s.g.) S. Then $x \in G$ can be extended to a (m.s.g.) of $G$ iff $h(x)=0 .\left(^{2}\right)$

REMARK. Before stating our next theorem, we remark that all basic subgroups of a primary group are isomorphic; and for nonprimary groups while I of the following theorem is implied by VI, I does not in general imply VI, as is the case in a group which is the direct sum of an infinite number of cyclic groups having different primes for orders.

THEOREM 1. Let $G$ be a primary p-group. Then the following statements are equivalent:

(I) G has a minimal system of generators.

(II) Some system of representatives of some basis of $G / p G$ generates $G$.

(III) $G$ is finite or $G / p G$ has the same power as $G$.

(IV) $G$ has the same power as a basic subgroup of itself.

(V) $G$ has a direct summand which is a direct sum of cyclic groups and has the same power as $G$.

(VI) $G$ has a minimum system of generators.

(VII) $G$ is finite or the automorphism group of $G / D$ has power equal to that of the set of all subsets of $G$, where $D$ is the divisible part of $G$.

(VIII) $G$ is finite or the automorphism group of $G / D$ has power exceeding that of $G, D$ being as in (VII).

Proof of (I) implies (II). Assume $G$ has a (m.s.g.) $S$. Then if $\sigma$ is the natural homomorphisin of $G$ onto $G / p G$, certainly the set $(\sigma(x))_{x \in S}$ generates $G / p G$, and it remains to show that this set is independent. So assume that for some $x \in S, \quad x+p G=\left(n_{1} x_{1}+p G\right)+\cdots+\left(n_{m} x_{m}+p G\right)$, where each $x_{i} \in S$, and

${ }^{2}$ Added in proof. Lemma 3 has an analogue for an arbitrary Abelian group $G$, in which case Lemma 1 is best proved by observing that $G /\{S \backslash(x)\}$ is cyclic. 
$x_{i} \neq x$. Then $\exists g_{1}, \cdots, g_{m} \in G$ such that $x=n_{1} x_{1}+\cdots+n_{m} x_{m}$ $+p\left(g_{1}+\cdots+g_{m}\right)$. Then by Lemma 2 the set $(S \backslash(x)) \cup\left(p\left(g_{1}+\cdots+g_{m}\right)\right)$ is a (m.s.g.) of $G$. This contradicts Lemma 1 since $h\left(p\left(g_{1}+\cdots+g_{m}\right)\right) \geqq 1$.

Next we prove (II) implies (III) implies (IV). If $G$ is finite there is nothing to prove. So assume $G$ is infinite; then since some system of representatives of some basis of $G / p G$ generates $G$, we must have $|G|=|G / p G|$. But by a theorem of Kulikov which holds for an arbitrary primary group $G$ and any basic subgroup $B$ of it we have $G / p G \cong B / p B$. Thus $|G|=|G / p G|$ $=|B / p B|$. Since $|G| \geqq|B| \geqq|B / p B|$, we obtain $|G|=|B|$. This completes the proof of (II) implies (III) implies (IV).

Proof of (IV) implies (V). Write $G=L \oplus D$ where $D$ is divisible and $L$ is reduced, and let $B$ be basic in $L$. Then $B$ is basic in $G$, so that $|G|=|B|$ $\leqq|L| \leqq|G|$. Thus we may assume that $G$ itself is reduced, since this would be the same as showing $L$ has a direct summand as desired.

We may assume that $G$ is infinite.

Case 1. $G$ is countable.

Any countable reduced $p$-group is known to have a direct summand which is a direct sum of a countable number of cyclic groups. See [1, p. 143].

Now let $B=\oplus_{i=1}^{\infty} B_{i}$, where $B_{i}$ is a direct sum of cyclic groups of order $p^{i}$, and $B$ is a basic subgroup of $G$.

Case 2. $|G|>\boldsymbol{\aleph}_{0}$, and $\exists$ an integer $n$ such that $\left|\oplus_{i=1}^{n} B_{i}\right|=|G|$.

It is then known that-see $\left[1\right.$, p. 98 , Baer's Theorem]-G $=\left(\oplus_{i=1}^{n} B_{i}\right)$ $\oplus\left\{\oplus_{i=n+1}^{\infty} B_{i}, p^{n} G\right\}$, and in this case we may take our direct summand to be $\oplus_{i=1}^{n} B_{i}$.

Case 3. $|G|>\boldsymbol{N}_{0}, G$ has no elements of infinite height, and for no integer $n$ is $\left|\oplus_{i=1}^{n} B_{i}\right|=|G|$.

Then there is no greatest $\left|B_{i}\right|$, and in this case $|B|=\sum_{i=1}^{\infty}\left|B_{i}\right|$, and $|B|$ is a "limit" cardinal. Let $i_{1}$ be the first integer such that $\left|B_{i_{1}}\right|$ is infinite, and assume $i_{n}$ has been defined. Let $i_{n+1}$ be the first integer larger than $i_{n}$ such that $\left|B_{i_{n+1}}\right|>\left|B_{i_{n}}\right|$. Then $\sum_{n=1}^{\infty}\left|B_{i_{n}}\right|=|B|=|G|$.

Now if we denote by $\bar{B}$ the closure of $B$, i.e., the torsion subgroup of the strong direct sum of the $B_{i}$ 's, then-since $G$ has no elements of infinite height -it is well known that $G$ may be considered as a pure subgroup of $\bar{B}$ - see $[1$, p. 112]. Then $G / B$ is divisible and of power not exceeding that of $B$. Let $S$ be a system of representatives of the nonzero cosets of $G / B$. Then each element of $S$ is a sequence $\left(v_{1}, v_{2}, v_{3}, \cdots\right)$ with $v_{i} \in B_{i}$, and where an infinite number of the $v_{i}$ 's are different from zero. Moreover, any element differing from $\left(v_{1}, v_{2}, v_{3}, \cdots\right)$ in only a finite number of places belongs to $G$ and represents the same coset of $G / B$ as $\left(v_{1}, v_{2}, v_{3}, \cdots\right)$.

Now $S$ may be divided into a sequence of disjoint subsets $S_{1}, S_{2}, S_{3}, \ldots$ such that $\left|S_{j}\right| \leqq\left|B_{i_{j}}\right|$, for $j=1,2,3, \cdots$.

By our last remark, we may assume in addition that each element of $S_{j}$ has its first $i_{j}$ components equal to zero. Now since each $\left|B_{i_{j}}\right|$ is infinite, 
every $B_{i_{j}}$ is a direct sum of cyclic groups $\oplus_{a \in A_{i_{j}}}\left\{v_{a}\right\}$ for some index set $A_{i_{j}}$ where $\left|A_{i_{j}}\right|=\left|B_{i_{j}}\right|$.

Now if $x \in S$ has some nonzero component from $B_{i_{j}}$, then $x \in S_{l}$ where $l<j$; moreover this component is of the form $k_{1} v_{a_{1}}+k_{2} v_{a_{2}}+\cdots+k_{m} v_{a_{m}}$ for some integers $k_{1}, \cdots, k_{m}$ and $a_{t} \in A_{i_{j}}$, for $t=1, \cdots, m$. Thus the set $C_{i_{j}}$ of all $a \in A_{i_{j}}$ such that $v_{a}$ occurs in an expression of a component of some element of $S$ is of cardinal $\leqq \aleph_{0}\left(\sum_{l<j}\left|B_{i_{l}}\right|\right)=\boldsymbol{N}_{0}\left|B_{i_{j-1}}\right|=\left|B_{i_{j-1}}\right|<\left|B_{i_{j}}\right|$.

Now $B_{i_{j}}=\left(\oplus_{a \in C_{i_{j}}}\left\{v_{a}\right\}\right) \oplus\left(\oplus_{a \in\left(A_{i_{j}} \backslash C_{i_{j}}\right)}\left\{v_{a}\right\}\right)$ where $\left|A_{i_{j}} \backslash C_{i_{j}}\right|=\left|B_{i_{j}}\right|$. Also $G$ is generated by $S$ and $\oplus_{i=1}^{\infty} B_{i}$. Moreover it is clear from the above that $G=\bigoplus_{j=1}^{\infty}\left(\oplus_{a \in\left(A_{i}, \backslash C_{i_{j}}\right)}\left\{v_{a}\right\}\right) \oplus\left\{S, \bigoplus_{j=1}^{\infty}\left(\oplus_{a \in C_{i j}}\left\{v_{a}\right\}\right)\right.$, all $B_{i}$ with $i \neq i_{j}$ for any $j\}$.

Thus $\oplus_{j=1}^{\infty}\left(\oplus_{a \in\left(A_{i j} \backslash C_{i_{j}}\right)}\left\{v_{a}\right\}\right)$ is a direct summand as required.

Case 4. $|G|>\aleph_{0}, G$ has elements of infinite height, and for no integer $n$ is $\left|\oplus_{i=1}^{n} B_{i}\right|=|G|$.

Let $\bar{G}$ be the subgroup of $G$ consisting of all the elements of $G$ of infinite height. Then by a theorem of Kulikov, $[1$, p. 103], the image $\bar{B}$ of $B$ under the natural homomorphism of $G$ onto $G / \bar{G}$ is a basic subgroup of $G / \bar{G}$ and $\bar{B} \cong B$. Now $\bar{B}$ and $G / \bar{G}$ satisfy the conditions of case 3 -see $[1$, p. 112]. Hence $G / \bar{G}$ may be written as $\bar{H} \oplus \bar{K}$ where $\bar{H}$ is a direct sum of cyclic groups, $|\bar{H}| \geqq|G / \bar{G}|$, and as in the proof of case $3, \bar{H}$ is contained in $\bar{B}$. Then let $H$ be the subgroup of $B$ generated by representatives from $B$ of the elements of $\bar{H}$. Then we have $(H+\bar{G}) / \bar{G}$ is a direct summand of $G / \bar{G}, H \cap \bar{G}=0$, and hence-see $[2$, p. 18$]-H$ is a direct summand of $G$. Then we have $|H|$ $=|\bar{H}| \geqq|G / \bar{G}| \geqq|\bar{B}|=|B|=|G|$. Hence $|H|=|G|$. Moreover, $H$ being a subgroup of $B$ which is a direct sum of cyclic groups, $H$ is also a direct sum of cyclic groups. This completes the proof of (IV) implies (V).

Proof of (V) implies (I). Let $G=H \oplus K$, where $|H| \geqq|K|$ and $H$ is a direct sum of cyclic groups. Then we must show that $G$ has a (m.s.g.). So let $B=\oplus_{a \in A}\left\{v_{a}\right\}$ be a basic subgroup of $K$. Then it is readily checked that $p B$ is a basic subgroup of $p K$ and $|p K / p B| \leqq|K| \leqq|H|$. Let $H=\oplus_{b \in Q}\left\{w_{b}\right\}$, and $p K / p B=\oplus_{z \in Z} D_{z}$, where each $D_{z}$ is an indecomposable divisible group. Then $Q$ may be divided into $|Z|+1$ sets $B_{z}, B_{1}$ such that for each $z \in Z$, $\left|B_{z}\right|=\boldsymbol{\aleph}_{0}$. Let $D_{z}$ be generated by $d_{1}^{2}, d_{2}^{2}, d_{3}^{2}, \cdots$ where $p d_{1}^{z}=0$, and $p d_{i+1}^{2}=d_{i}^{z}$ Let $e_{i}^{z} \in p K$ represent $d_{i}^{2}$. Now if each set $\left(w_{b}\right)_{b \in B_{z}}$ is arranged in a sequence $w_{1}^{2}$, $w_{2}^{z}, w_{3}^{2}, \cdots$ where the order of $w_{i}^{z}$ is $p^{n_{i}{ }^{2}}$, then for each $z \in Z$ form the set

$$
E_{z}=\left(w_{1}^{2}+e_{n_{1}}^{z}, w_{2}^{2}+e_{n_{1}{ }^{2} n_{2}}^{2}, \cdots, w_{m}^{2}+e_{n_{1}{ }^{2}+\cdots+n_{n}{ }^{2}}^{2}, \cdots\right) .
$$

Then we assert that the set

$$
T=\left(\bigcup_{a \in A}\left(v_{a}^{\prime}\right)\right) \cup\left(\bigcup_{b \in B_{1}}\left(w_{b}\right)\right) \cup\left(\bigcup_{z \in Z} E_{z}\right)
$$

is a (m.s.g.) of G. First we observe that the images of the elements of 


$$
\left(\underset{b \in B_{1}}{\bigcup}\left(w_{b}\right)\right) \cup\left(\underset{z \in Z}{\bigcup} E_{z}\right)
$$

under the natural homomorphism of $H+p K$ onto $(H+p K) / p B$ generate $(H+p K) / p B$, and that $\bigcup_{a \in A}\left(v_{a}\right)$ generates $B$ which contains $p B$. Thus $\{T\} \supset\{H+p K, B\}$. Now $\{p K, B\}=K$-see $[1$, p. 109]. Thus $\{T\}=G$. To show $T$ is minimal, we first observe that $\left\{T \backslash\left(w_{b}\right)\right\}$ does not contain $w_{b}$ for any $b \in B_{1}$, and that $\left\{T \backslash\left(w_{m}^{2}+e_{n_{1}}^{z}+\cdots+n_{m} s\right)\right\}$ does not contain $w_{m}^{2}$ for any $z \in Z$ and any integer $m$. Moreover we will show that for no $a_{0} \in A$ does $\left\{T \backslash\left(v_{a_{0}}\right)\right\}$ contain $v_{a_{0}}$.

The last assertion follows from: $\left\{T \backslash\left(v_{a_{0}}\right)\right\} \subset\left\{H, p K,\left(v_{a}\right)_{a_{0} \neq a \in A}\right\}$ which does not contain $v_{a_{0}}$, since the images of the $v_{a}$ 's are independent in $G /(H+p K)$, as mentioned above. This completes the proof of (V) implies (I).

Proof of (I) is equivalent to (VI). We will prove the stronger statement, namely, $S$ is a (m.s.g.) of $G$ iff $S$ is a (M.s.g.) of $G$. It follows at once from the definitions that if $S$ is a (M.s.g.) of $G$, then $S$ is a (m.s.g.) of $G$.

Next assume that $S$ is a (m.s.g.) of $G$, and that for some $n \geqq 1$ the $n$ elements $x_{1}, \cdots, x_{n}$ of $S$ may be replaced by the $n-1$ elements $y_{1}, \cdots, y_{n-1}$ of $G$ in such a way that the resulting set $Q$ still generates $G$. Hence for each $x_{i} \exists x_{1}^{i}, \cdots, x_{m_{i}}^{i} \in S \backslash\left(x_{1}, \cdots, x_{n}\right)$ such that $x_{i} \in\left\{x_{1}^{i}, \cdots, x_{m}^{i}, y_{1}, \cdots, y_{n-1}\right\}$.

Consider the subset

$$
T=\left(x_{1}, \cdots, x_{n}\right) \cup\left(\bigcup_{i=1}^{n}\left(x_{1}^{i}, \cdots, x_{m_{i}}^{i}\right)\right)
$$

of $S . T$ is finite and consists, say, of $k$ elements. Hence $Q=\left(T \backslash\left(x_{1}, \cdots, x_{n}\right)\right)$ $\cup\left(y_{1}, \cdots, y_{n-1}\right)$ has $k-1$ elements. If $\sigma$ is the natural homomorphism of $G$ onto $G / p G$, this implies that $\sigma(T)$ which by the proof of (I) implies (II) forms part of a basis of $G / p G$ consisting of $k$ elements is dependent in $G / p G$ on the $k-1$ elements $\sigma(Q)$ which is impossible. This contradiction proves our assertion.

Finally, we prove that (VII) is equivalent to (V).

Assume that $G$ is infinite, and that it has a direct summand as specified in $(\mathrm{V})$. Then it is easy to verify that the power of the automorphism group of $G$ is equal to $2^{|G|}$. Next assume (V) is not satisfied. If $|G|>|G / D|$ the conclusion is obvious. If not, we may assume $G$ is reduced, and by the equivalence of (IV) and (V), the power of $G$ would exceed that of any of its basic subgroups. Then an unpublished theorem of $\mathrm{E}$. Walker, delivered to the author by oral communication, see $[4$, p. 867$]$, asserts that the power of the automorphism group of $G$ is $|G|$. This also proves that (V) is equivalent to (VIII).

With this the proof of Theorem 1 is complete.

REMARK. From the proof of the equivalence of (I) and (IV) of Theorem 1, Lemma 3 can be stated as follows:

Lemma 3a. Let $G$ have a (M.s.g.). Then $x \in G$ can be extended to a (M.s.g.) of $G$ iff $h(x)=0$. 
Definition. Let $G$ be called a starred group if it satisfies any one of the eight equivalent conditions of Theorem 1. The following corollaries are obtained from Theorem 1 using well-known properties of abelian groups.

CoRollary 1. A primary divisible group $G$ is not a starred group.

Corollary 2. Let $B$ be basic in $G$, and $H \supset B$ be a subgroup of $G$. Then if $|H|=|B|, H$ is starred. Further, if $H$ is pure in $G$, then $H$ is starred only if $|H|=|B|$. In particular, if $G$ itself is starred then any subgroup of $G$ containing $B$ is starred.

COROLlaRy 3. There exists a primary group without elements of infinite height which is not starred.

Corollary 4. Any countable reduced primary group is starred.

Corollary 5. Let $G$ be a reduced primary group and $B$ be basic in $G$. Assume $|B|=|B| \aleph_{0}$. Then $G$ is starred.

COROLlaRy 6. Let $G$ be reduced, and such that $n^{\aleph_{0}}<|G|$ for any cardinal $n$ less than $|G|$. Then $G$ is starred.

CoRollary 7. Let $G$ be infinite. Then $G$ is starred iff the reduced part of $G$ is the direct sum of $|G|$ nontrivial subgroups of $G$.

L. Fuchs asks the following question-see [1, Problem 18, p. 144]:

"Which are the cardinals $m$ such that there exist no $m$-indecomposable reduced $p$-groups (i.e., primary groups) of power $m$ ?"

We have

THEOREM 2a. There exists no infinite $m$-indecomposable reduced primary group of power $m$ if and only if either $m=\mathbf{N}_{0}$ or for every cardinal $n<m$ we have $n^{\aleph_{0}}<m$.

Since by Corollary 7 for reduced groups of infinite cardinal $m, m$-decomposability is equivalent to the property of being starred, we may prove instead the following version of Theorem $2 \mathrm{a}$ :

THEOREM 2. There exists an unstarred reduced group $G$ of infinite cardinal $m$ if and only if there exists an infinite cardinal $n<m$ such that $n \aleph_{0} \geqq m$.

Proof. If $m=\boldsymbol{\aleph}_{0}$, Corollary 4 implies that $G$ is starred. If $m>\boldsymbol{\aleph}_{0}$ and for no infinite cardinal $n<m$ is $n^{\aleph_{0}} \geqq m$, then Corollary 6 implies that $G$ is starred.

Next assume $m$ is a cardinal for which there exists an infinite cardinal $n<m$ such that $n^{\aleph_{0}} \geqq m$. Then $m>\aleph_{0}$. Let $B_{i}$ be the direct sum of $n$ cyclic groups of order $p^{i}$. Let $\bar{B}$ be the closure of $B=\oplus_{i=1}^{\infty} B_{i}$. Then $|B|=n \boldsymbol{\aleph}_{0}, \bar{B}$ is reduced (and even has no elements of infinite height) and $|\bar{B}|=n \boldsymbol{\aleph}_{0} \geqq m>n \aleph_{0}$. Also $\bar{B} / B$ is divisible and of cardinal $\geqq m$ since $|B| \leqq n \aleph_{0}$. Then there exists a pure subgroup $B^{*} / B$ of $\bar{B} / B$ (where $B^{*} \supset B$ ) of cardinal $m$. Then $\left|B^{*}\right|=m$, and $B$ is basic in $B^{*}$, which by IV of Theorem 1 means $B^{*}$ is not starred. 
This concludes the proof of Theorem 2.

Problem 14 in L. Fuchs' book Abelian groups reads as follows: "Under what conditions can every element of a $p$-group be embedded in a direct summand of power $\leqq m, m$ an infinite cardinal?"

In this connection we have

THEOREM 3. Let $G$ be an infinite starred group, and let $x \in G$ be contained in some pure subgroup of $G$ having no elements of infinite height. Then $x$ can be embedded in a direct summand $H$ of $G$ of power $m$ for any $m$ satisfying $m \leqq|G|$.

Proof. It is known that such an $x$ can be embedded in a finite direct summand $K$ of $G$. Then let $G=K \oplus L$, and let $B$ be a basic subgroup of $L$. Then $|B|=|L|$, and by IV of Theorem $1 L$ is starred. By V of Theorem $1, L=L_{1} \oplus L_{2}$ where $\left|L_{1}\right|=|L|=|G|$ and $L_{1}$ is a direct sum of cyclic groups. If $|G| \geqq m$, then clearly $L_{1}$ has a direct summand $L_{3}$ satisfying $\left|L_{3}\right|=m$. We may then set $H=K \oplus L_{3}$.

REMARK. If any $x \in G$ which is contained in some pure subgroup of $G$ having no elements of infinite height is also contained in some direct summand of $G$ having power $m$ for any $m \leqq|G|$, then it does not follow that $G$ is starred.

To see this we let $G$ be the direct sum of a countable number of groups each isomorphic to $\bar{B}$ of the proof of Theorem 2 with $n=1$.

The following theorem is included for the sake of completeness:

THEOREM 4. Let $G$ be a primary p-group. Then $G$ is a direct sum of cyclic groups of bounded order if and only if there is some basis of $G / p G$ for which any system of representatives by elements of $G$ generates $G$. If every system of representatives of some basis of $G / p G$ generates $G$, then every system of representatives of every basis of $G / p G$ generates $G$.

Proof. Assume first that $G=\oplus_{i=1}^{n} B_{i}$ where each $B_{i}$ is a direct sum of cyclic groups $\left\{v_{a_{i}}\right\}_{a_{i} \in A_{i}}$ of order $p^{i}$, and $n$ is an integer. For any primary $p$-group $H$, let $S(H)$ denote the subgroup of $H$ consisting of those elements of $H$ whose orders are $p$ or 1 . For any subgroup $K$ of $G$ let $K^{i}=p^{i} K$. Then $0=G^{n} \subset G^{n-1}$ $\subset \cdots \subset G^{0}=G$, and $G^{i}=B_{i+1}^{i} \oplus \cdots \oplus B_{n}^{i}$. Also let $\sigma_{i}$ be the natural homomorphism of $G$ onto $G / G^{i}$. Then $\left(\sigma_{1}\left(v_{a_{2}}\right)\right.$, all $a_{i} \in A_{i}$ and $\left.i=1, \cdots, n\right)$ is a basis of $G / p G$, and any system of representatives of it is of the form $L=\left(v_{a_{i}}+p\left(g_{a_{i}}\right)\right.$, all $a_{i} \in A_{i}$ and $i=1, \cdots, n$, and where $g_{a_{i}}$ is some element of $G)$. We will show that $\{L\} \supset S(G)=G^{n-1}=B_{n}^{n-1}$. Let $x \in B_{n}^{n-1}$; then $\exists v_{a_{1} n}, \cdots, v_{a_{m} n}$ and integers $l_{1}, \cdots, l_{m}$ such that $x=l_{1} v_{a_{1} n}+\cdots+l_{m} v_{a_{m} n}$ where $p^{n-1}$ divides each $l_{i}$. Then also

$$
x=l_{1}\left(v_{a_{1} n}+p\left(g_{a_{1} n}\right)\right)+\cdots+l_{m}\left(v_{a_{m} n}+p\left(g_{a_{m} n}\right)\right)
$$

since $p^{n} y=0$ for any $y \in G$. Thus $x \in\{L\}$. Thus $\{L\} \supset B_{n}^{n-1}=G^{n-1}$. Similarly $\left\{\sigma_{n-1}(L)\right\} \supset S\left(G / G^{n-1}\right)$. Thus $\{L\} \supset G^{n-2}$. In the same way, having shown 
that $\{L\} \supset G^{m}$, it follows that $\left\{\sigma_{m}(L)\right\} \supset S\left(G / G^{m}\right)$, and hence that $\{L\}$ $\supset G^{m-1}$. After a finite number of steps we arrive at $\{L\} \supset G^{0}=G$. Thus if $G$ is a bounded direct sum of cyclic groups, then there is a basis of $G / p G$ such that any system of representatives of this basis generates $G$.

Next we assume that there is a basis $\left(\bar{v}_{a}\right)_{a \in A}$ of $G / p G$ for which any system of representatives generates $G$, and we prove that if $\left(\bar{w}_{b}\right)_{b \in B}$ is any other basis of $G / p G$ then any system of representatives $\left(w_{b}\right)_{b \in B}$ of it generates $G$. If $\bar{v}_{a}=l_{n}^{a} \bar{w}_{b_{1}}+\cdots+l_{n}^{a} \bar{w}_{b_{n}}$, then $l_{1}^{a} w_{b_{1}}+\cdots+l_{n}^{a} w_{b_{n}}$ is certainly a representative of $\bar{v}_{a}$. Then the set $\left(l_{1}^{a} w_{b_{1}}+\cdots+l_{n}^{a} w_{b_{n}}\right)_{a \in A}$ generates $G$. Hence $\left(w_{b}\right)_{b \in B}$ also generates $G$.

Now we prove that if there is some basis of $G / p G$ for which any system of representatives generates $G$, then $G$ is a bounded direct sum of cyclic groups. By what we have just proved, any system of representatives of any basis of $G / p G$ generates $G$. Let $B=\bigoplus_{a \in A}\left\{v_{a}\right\}$ be a basic subgroup of $G$. Then it is well known that if $\sigma$ is the natural homomorphism of $G$ onto $G / p G,\left(\sigma\left(v_{a}\right)\right)_{a \in A}$ is a basis of $G / p G$. If we let $v_{a}$ represent $\sigma\left(v_{a}\right)$ we obtain that $G=B$, or that $G$ is a direct sum of cyclic groups. We must still show that $G$ is bounded. If $G$ were not bounded, there would exist $a_{1}, a_{2}, a_{3}, \cdots \in A$ such that order $v_{a_{i}}=p^{n_{i}}$, where $n_{i+1}>n_{i}$ for $i=1,2,3, \cdots$. Then $\left(v_{a}, a \in A, a \neq a_{i}\right.$ for any $\left.i\right)$ $\cup\left(v_{a_{i}}+p^{n_{i+1}-n_{i}} v_{a_{i+1}}, i=1,2,3, \cdots\right)$ certainly represents a basis of $G / p G$, and as is well known-this is also easy to see-does not generate $G$. The proof of Theorem 4 is now complete.

\section{On torsion groups.}

Notation. Let $P$ denote the set of primes.

Definition. If $T$ is an Abelian torsion group and $p \in P$, let $T_{p}$ be the $p$-component of $T$. A subgroup $B$ of $T$ is said to be a basic subgroup of $T$ if and only if $B_{p}$ is a basic subgroup of $T_{p}$ for every $p \in P$.

TheOREm 5. Let $T$ be an Abelian torsion group. Then the following statements are equivalent.

(I) $T$ has a minimal system of generators.

(II) $T$ has the same power as a basic subgroup of itself.

(III) $T$ has a direct summand which is a direct sum of cyclic groups and has the same power as $T$.

Proof. First we prove that I implies II. So let $B=\bigoplus_{a \in A}\left\{v_{a}\right\}$ be a basic subgroup of $T$, and recall that all basic subgroups of a group are isomorphic. Let $S$ be a (m.s.g.) of $T$. With each $v_{a}$ associate a finite subset $S_{a}$ of $S$ such that $v_{a} \in\left\{S_{a}\right\}$. If we now assume that $|B|<|T|$, we obtain that $B \subset\left\{\cup_{a \in A} S_{a}\right\}$ $<T$. Then if $\sigma$ is the natural homomorphism of $T$ onto $T /\left\{\bigcup_{a \in A} S_{a}\right\}$, $\sigma\left(S \backslash \bigcup_{a \in A} S_{a}\right)$ is a (m.s.g.) of $T /\left\{\bigcup_{a \in A} S_{a}\right\}$ which is divisible since it is a homomorphic image of $T / B$, and $T / B$ is divisible since each $T_{p} / B_{p}$ is. Thus it remains to show that no (non-zero) divisible torsion group $D$ has a (m.s.g.) $Q$. Let $x \in Q$; then $D>\{Q \backslash(x)\}$, and $\sigma(x)$ is a (m.s.g.) of $D /\{Q \backslash(x)\}$ where $\sigma$ 
is the natural homomorphism of $D$ onto $D /\{Q \backslash(x)\}$. But $D /\{Q \backslash(x)\}$ cannot be generated by one element since being a homomorphic image of $D$ it is itself divisible. Thus I implies II.

Trivially III implies II.

Next we assume that $|T|=|B|$ for some basic subgroup $B$ of $T$ and show that $T$ has a (m.s.g.), showing in the process that II implies III, i.e., we go from II to I via III.

Case 1. For each prime $p, B_{p}$ is finite. If $B_{p} \neq 0$, for only a finite number of primes $p_{1}, \cdots, p_{n}$, then $T=\oplus_{i=1}^{n} B_{p_{i}}$, and the result follows. In the other case, $T_{p}=B_{p} \oplus D_{p}$ where $D_{p}$ is divisible. Then necessarily $\boldsymbol{\aleph}_{0}=\left|\oplus_{p \in P} B_{p}\right|$ $\geqq\left|\oplus_{p \in P} D_{p}\right|$. Let $\oplus_{p \in P} B_{p}=\oplus_{a \in A}\left\{v_{a}\right\}$, where the $v_{a}$ 's have prime power order. Then since for each $p \in P,\left|B_{p}\right|<\boldsymbol{\aleph}_{0}$, we may map the elements of prime power order of $\oplus_{p \in P} D_{p}$ in a one-one fashion on a subset $\left(v_{a}\right)_{a \in B \subset A}$ of $\left(v_{a}\right)_{a \in A}$ such that no element of $D_{p}$ is mapped on an element of $B_{p}$. For a justification of this see the proof of case 3 . Then the set $\left(v_{a}+w_{a}\right)_{a \in A}$ where $w_{a}$ is the element mapped on $v_{a}$ if there is such a one, and $w_{a}$ is zero otherwise is easily seen to be a (m.s.g.) of $T$.

Case 2. For some $p_{0} \in P$, there is an infinite $B_{p_{0}}$ with $\left|B_{p_{0}}\right|=|T|$. In this case $\left|B_{p_{0}}\right|=\left|T_{p_{0}}\right|$, and by $\mathrm{V}$ of Theorem $1, T_{p_{0}}=T^{1} \oplus T^{2}$ where $T^{1}$ is an infinite direct sum of cyclic groups $\oplus_{a \in A}\left\{v_{a}\right\}$, and $|T|=\left|T^{1}\right| \geqq\left|T^{2}\right|$. Then we may write $A$ as the union of two disjoint subsets $A_{1}$ and $A_{2}$ with $\left|A_{1}\right|=\left|A_{2}\right|$ $=|A|=|T|$. By V of Theorem 1, $T^{2} \oplus\left(\oplus_{a \in A_{1}}\left\{v_{a}\right\}\right)$ has a (m.s.g.) $S$. Then we may map the elements of $\oplus_{p_{0} \neq p \in P} T_{p}$ in a one-one fashion on a subset of $\left(v_{a}\right)_{a \in A_{z}}$. Then the set $S \cup\left(v_{a}+w_{a}\right)_{a \in A_{z}}$ where $w_{a}$ is the element mapped on $v_{a}$ if there is such a one and $w_{a}$ is zero otherwise is easily seen to be a (m.s.g.) of $T$, and even a (M.s.g.) of $T$.

Case 3. Neither Case 1 nor Case 2 holds. Then there is no greatest $\left|B_{p}\right|$. For each $p \in P$, let $B_{p}=\oplus_{i=1}^{\infty} B_{p}^{i}$, where $B_{p}^{t}$ is a direct sum of cyclic groups of order $p^{i}$. Then one can verify that there is a subsequence $p_{1}, p_{2}, p_{3}, \cdots$ of the sequence of primes and integers $i_{1}, i_{2}, i_{3}, \cdots, i_{j}$ depending on $P_{j}$ such that $\sum_{j=1}^{\infty}\left|B_{p_{i}}^{i_{j}}\right|=|T|$, and $\left|B_{p_{j}}^{i_{j}}\right|<\left|B_{p_{j+1}}^{i_{j+1}}\right|$ for $j=1,2,3, \cdots$. Since each $B_{p}^{i}$ is a direct summand of $T_{p}$, we have $T^{1+1}=T^{1} \oplus \oplus_{j=1}^{\infty} B_{p}^{i_{j}}$. Let $\oplus_{j=1}^{\infty} B_{p_{j}}^{t_{j}}=\oplus_{a \in A}\left\{v_{a}\right\}$, where each $v_{a}$ belongs to some $B_{p}$. Divide $A$ into a sequence of disjoint subsets $A_{1}, A_{2}, A_{3}, \cdots$ such that $\left|A_{k}\right|=|A|=|T|$ for each integer $k$. Then for each integer $k,\left|\left(v_{a}\right)_{a \in A_{k}} \cap T_{p_{k}}\right|<|T|$. Thus if $q_{1}, q_{2}, q_{3}, \cdots$ is the sequence of primes, we may map the elements of $T_{q_{l}}^{1}$ in a one-one fashion onto a subset of $\left(\left(v_{a}\right)_{a \in A_{l}} \backslash T_{q_{l}}\right)$. Then the set $\left(v_{a}+w_{a}\right)_{a \in A}$ where $w_{a}$ is the element mapped on $v_{a}$ if there is such a one and $w_{a}$ is zero otherwise can be verified to be a (m.s.g.) of $T$.

This completes the proof of Theorem 5 .

THEOREM 6. Let $T$ be an Abelian torsion group. Then the following statements are equivalent:

(I) $T$ has a minimum system of generators. 
(II) $T$ is finite or some basic subgroup of some primary component of $T$ has the same power as $T$.

(III) $T$ is finite or $T$ has a direct summand of the same power as $T$ which is a direct sum of cyclic groups whose orders are powers of the same prime.

Proof. The theorem is easily verified for finite groups. Thus we may assume in the following that $T$ is infinite. First we prove that I implies II. So assume that for no $p \in P$ is $\left|B_{p}\right|=T$, and let $S$ be a (M.s.g.) of $T$. Let $Q$ be the set of primes such that $\left|T_{q}\right|<|T|$ for each $q \in Q$. For each $x \in T$, let $x=x_{p_{1}}+\cdots+x_{p_{n}}$, where $x_{p_{i}} \in T_{p_{i}}$, the $p_{i}$ 's being different primes, and let $P_{x}=\left(p_{1}, \cdots, p_{n}\right)$. Then we assume that $\mid\left(x \in S\right.$ such that $\left.p \in P_{x}\right)|\leqq| T_{p} \mid$ for each $p \in Q$. Now let $x \in S$ be written as $x=x_{p_{1}}+\cdots+x_{p_{n}}$ as above. Then we may assume that $\left(P_{1}, \cdots, P_{m}\right) \subset P \backslash Q$, and $\left(P_{m+1}, \cdots, P_{n}\right) \subset Q$ for some integer $m$. Now since for $P_{m+j}, j=1, \cdots, n-m,\left|T_{p_{m+j}}\right|<|T|$, there exist $n-m$ different elements $y_{(m+j)} \in S$ with $y_{p_{m+j}}=0$, where the index $(m+j)$ in brackets does not mean that $y_{(m+j)} \in T_{m+j}$. Let $\bar{y}_{(m+j)}=y_{(m+j)}+x_{p_{m+j}}$. Moreover, since for $i=1, \cdots, m,\left|B_{p_{i}}\right|<|T|$, and $\left|T_{p_{i}}\right|=|T|$, by Theorem 1 $T_{p_{i}}$ has no (m.s.g.). But $T_{p_{i}} \subset\left\{\left(x_{p_{i}}\right)_{x \in S}\right\}$. Thus there exist $m$ different elements $y_{\left(p_{i}\right)} \in\left(S \backslash\left(y_{(m+1)}, \cdots, y_{(n)}, x\right)\right)$ such that for $i=1, \cdots, m, T_{p_{\boldsymbol{i}}}$ $C\left\{\left(x_{p_{i}}\right)_{y_{\left(p_{i}\right)} \neq x \in S}\right\}$, where the index in brackets of $y_{\left(p_{i}\right)}$ does not mean that $y_{\left(p_{i}\right)} \in T_{p_{i} \cdot}$ Then if $y_{\left(p_{i}\right)}=y_{p_{1}}+\cdots+y_{p_{i}}+\cdots+y_{p_{k}}$, let $y_{(i)}=y_{p_{1}}+\cdots$ $+y_{p_{i-1}}+x_{p_{i}}+y_{p_{i+1}}+\cdots+y_{p_{k}}$.

Then the set

$\left[\left(y_{(1)}, \cdots, y_{(m)}, \bar{y}_{(m+1)}, \cdots, \bar{y}_{(n)}\right)\right.$

$$
\left.\cup\left(S \backslash\left(x, y_{\left(p_{1}\right)}, \cdots, y_{\left(p_{m}\right)}, y_{(m+1)}, \cdots, y_{(n)}\right)\right)\right]
$$

is easily seen to generate $G$. But this set was obtained from $S$ by replacing $n+1$ elements of $S$ by $n$ elements of $G$. This contradicts the minimum property of $S$. Thus I implies II. The proof of II implies III follows easily from Theorem 1. The proof of III implies I is contained in Case 2 of the proof of Theorem 5-see the last sentence of the proof of that case.

The following theorem is partly included in results obtained by $W$. R. Scott, $[5$, p. 19-22], and is included here as an illustration of the applicability of Theorems 5 and 6.

Theorem 7. Let $T$ be an Abelian torsion group having a minimal system of generators and having one infinite primary component. Then $T$ has $2^{|T|}$ different subgroups all isomorphic to $T$; for every cardinal $n \leqq|T|, T$ has $2^{n}$ different isomorphic subgroups of cardinal $n ; T$ has $2^{|T|}$ different automorphisms mapping some fixed basic subgroup of $T$ onto itself, and $T$ has $2^{|T|}$ different direct summands.

Proof. By III of Theorem 5, $T=H \oplus K$ where $|H|=|T|$, and $H$ is a direct sum of cyclic groups. 
To prove the first part, it is easy to establish, because of the particularly simple structure of $I I$, that for any $n \leqq|T|, H$ has $2^{n}$ different isomorphic subgroups $\left(H_{a}\right)_{a \in A}$ of power $n$, all isomorphic to $H$ if $n=|T|$. If $n=|T|$, then $\left(H_{a}+K\right)_{a \in A}$ are the desired $2^{|T|}$ different subgroups isomorphic to $T$.

To prove the second part, let $B$ be a basic subgroup of $K$. Again because of the simple structure of $H$, it is easy to establish that $H$ has $2^{|T|}$ different automorphisms $\left(\sigma_{a}\right)_{a \in A}$. Let $\bar{\sigma}_{a}$ be the automorphism of $T$ which coincides with $\sigma_{a}$ on $H$ and maps $K$ identically on itself. Then $\left(\bar{\sigma}_{a}\right)_{a \in A}$ is a set of $2^{|\boldsymbol{T}|}$ automorphisms of $T$ all of which map $H \oplus B$ which is a basic subgroup of $T$ onto itself. The proof of the last statement is obvious.

THEOREM 8. Let $G$ be an infinite reduced primary group, and $B$ be a basic subgroup of $G$. Then the power of the automorphism group of $G$ is $2^{|B|}$.

Proof. E. Walker proved this result in the case $|B|<|G|$; see $[4$, p. 867]. If $|B|=|G|$, then Theorem 7 applies.

Theorem 9. Let $T$ be an Abelian torsion group. Then $T$ can be embedded as a direct summand in an Abelian torsion group $H$ with a minimum system of generators (which is a fortiori a minimal system of generators) satisfying $|H|$ $=|T|$.

Proof. If $T$ is finite, set $H=T$. If $T$ is infinite, then let $K$ be a direct sum of $|T|$ finite cyclic groups whose orders are powers of the same prime. Set $H=T \oplus K$. Theorem 6 completes the proof.

Theorem 10. Let $T$ be an Abelian torsion group. Then $T$ can be embedded as a fully invariant subgroup in a group $H$ having a minimum system of generators (which is a fortiori a minimal system of generators) and having the same cardinal as $T$; more precisely, if $T$ is infinite, then $T$ can be embedded as a fully invariant subgroup in a group $H$ having a minimum system of generators such that $T=n H$ for any prescribed integer $n>1$.

Proof. If $T$ is finite set $H=T$. If $T$ is infinite we proceed as follows: There exists a free Abelian group $F=\oplus_{a \in A}\left\{v_{a}\right\}$ with $|F|=|T|$ and a subgroup $K$ of $F$ such that $F / K \cong T$. Consider another set $\left(w_{a}\right)_{a \in A}$ of free generators. Identify each $v_{a}$ with $n w_{a}$, and thus $F$ with $n\left\{\left(w_{a}\right)_{a \in A}\right\}$. Let $L=\left\{\left(w_{a}\right)_{a \in A}\right\} / K$. Then clearly $n L=T$, and $|L|=|T|$. Now let $p$ be a prime dividing $n$, and let $Z$ be a direct sum of $|T|$ cyclic groups of order $p$. Set $H=Z \oplus L$. Then $|H|=|T|$, and by Theorem $6 H$ has a (M.s.g.). Then also $n H=n Z \oplus n L$ $=n L=T$, which implies the full invariance of $T$ in $H$.

CoRollary 8. Let $G$ be a primary group. Then $G$ can be embedded as a direct summand in a starred (primary) group $H$ satisfying $|H|=|T|$.

Corollary 9. Let $G$ be a p-group. Then $G$ can be embedded as a fully invariant subgroup in a starred group $H$ of the same cardinal, more precisely; if $G$ is 
infinite then $G$ can be embedded as a fully invariant subgroup in a starred group $H$ of the same cardinal such that $G=p H$.

REMARK. If in Corollary $8, H=K \oplus G$ where $K$ is a direct sum of cyclic groups of order $p$, then this embedding coincides with that of Corollary 9 in the sense that $p H=G$ if and only if $G$ is divisible, for $G=p H=p K \oplus p G=p G$, implies that $G$ is divisible.

REMARK. W. R. Scott suggested generalizing the above results to modules over countable rings. It turns out that most of the above theorems and in particular Theorem 1 hold for torsion modules over a principal ideal ring or over a complete discrete valuation ring, after the customary changes in terminology - such as substituting finitely generated for finite, etc.-are made; see $[1$, p. 29].

\section{BIBLIOGRAPHY}

1. L. Fuchs, Abelian groups, Budapest, Hungarian Academy of Sciences, 1958. 1954.

2. Irving Kaplansky, Infinite Abelian groups, Ann Arbor, University of Michigan Press,

3. A. G. Kurosh, The theory of groups, New York, Chelsea Publishing Company, 1955.

4. E. A. Walker, Abstract 564-235, Notices Amer. Math. Soc. vol. 6 (1959) p. 867.

5. W. R. Scott, The number of subgroups of given index in non-denumerable Abelian groups, Proc. Amer. Math. Soc. vol. 5 (1954) pp. 19-22.

UNIVERSITY OF KANSAS,

LAWRENCE, KANSAS 